\title{
Risikominimierung der Keim-Reinfektion nach parodontalchirurgischen Eingriffen
}

Wie wichtig die frühe Therapie von Gingivitis als mögliche Vorstufe der Parodontitis - ist, wurde im Juni 2015 in London von führenden Experten auf dem EuroPerio 8 erläutert. Im Rahmen eines Symposiums für Zahnärzte und Parodontologen, veranstaltet von der GSK Consumer Healthcare, wurde die Reduzierung der Gingivitis als eines der präventiven Hauptziele in der zahnärztlichen Praxis erklärt. Schreiten Gingivitis und - möglicherweise in Folge - Parodontitis fort, sind unter Umständen parodontalchirurgische Maßnahmen nötig. Chlorhexidinhaltige Produkte mit einer Konzentration von 0,2\% Chlorhexidine (CHX) gelten nach PA-Be- handlungen als Goldstandard. Zahnärzte können sich auf den sicheren und bewährten Wirkmechanismus von Chlorhexidin verlassen. CHX gilt im Risikomanagement und in der Infektionsbekämpfung nach parodontalchirurgischen Eingriffen als Goldstandard. Auf der EuroPerio 8 empfahlen $40 \%$ der Teilnehmer des GSKSymposiums die Anwendung einer antiseptischen Mundspülung zur Behandlung einer Gingivitis, 53\% empfahlen konkret ein Chlorhexidin-haltiges Produkt.

Nach einer Pressemitteilung der GlaxoSmithKline Consumer Healthcare $\mathrm{GmbH}$ \& Co. KG, Bühl 